\title{
Surgery-First Orthognathic Approach
}

\author{
N. Viveka Vardhan Reddy and Abhinand Potturi
}

\subsection{Introduction}

Treatment philosophies for the management of dentofacial deformities are varied. Milder forms of dentofacial deformity not manifesting as a significant skeletal discrepancy can be corrected by orthodontic treatment alone called "orthodontic camouflage", but those presenting with significant skeletal discrepancy need the skills of both the orthodontist and the maxillofacial surgeon. Hence, correction of dentofacial deformities has come to be a confederate work between both specialties. The dentition and maxillomandibular skeletal complex (MMSC) are intertwined, and changes to one will invariably affect the other, especially more so when the MMSC positional and spatial relationships change. Over the years many new concepts have evolved in orthognathic surgery, and the odyssey between the surgical and orthodontic teams is only getting more inseparable and interesting.

\subsection{Background}

Looking back to 1960 s when techniques of mandibular and maxillary orthognathic surgeries were still evolving from the works of Obwegeser and Trauner, surgeons often performed the procedures either before orthodontic treatment or well after the completion of orthodontic treatment, and rarely any surgeon depended on an orthodontist to move the teeth into certain relationship before undertaking surgery [1]. With time it was realized that the natural dental compensations became a hindrance to the optimal movement of MMSC, thus compromising the results and final outcome.

Poulton et al. [1] in 1963 reported few cases of excessive mandibular growth (mandibular prognathism) which were treated surgically without any pre-surgical orthodontics. In the process, the authors observed that the overjet relationship between the upper and lower anterior teeth limited the amount of mandibular setback, thereby compromising the overall treatment outcome. They concluded that proper alignment of teeth in upper and lower arches is a prerequisite to get adequate mandibular setback. This proposal had almost paved the way for the "orthodontics-first" concept for correcting dentofacial deformities. Worms et al. [2] in the 1970s popularized the concept of "orthodontics first" for all orthognathic surgeries which led to the split of orthodontic treatment into two phases: the pre-surgical orthodontics phase and post-surgical orthodontics phase with an intervening surgical phase.

The pre-surgical orthodontics phase, which essentially antecedes the orthognathic surgical phase, brings out the accurate skeletal discordance by decompensating the natural compensations which have occurred, thereby helping the surgeon to perform a more precise spatial relocation of the MMSC during surgery [3]. However, the pre-surgical phase of orthodontic treatment, also called reverse orthodontics, is a long-drawn process lasting anywhere between 1 and 2 years depending on the complexity of the discrepancy [4]. To add to the woes, during this phase there is gradual deterioration of facial form and dental function [5-7]. Worsening of the facial profile has become a great deterrent for the patients seeking orthognathic surgery because the very reason for seeking the orthognathic surgery for improving facial aesthetics stands defeated and therefore fails to address the patients' chief complaint. Also, the long preoperative preparatory phase can aggravate or initiate other dental problems like dental caries or periodontal problems. For all these reasons, the "orthodontics-first" concept can produce a negative influence on the patient's compliance [8] (Table 67.1).

N. V. V. Reddy $(\varangle) \cdot$ A. Potturi

SVS Institute of Dental Sciences, Mahabubnagar, Telangana, India 
Table 67.1 Challenges associated with conventional "orthodonticsfirst" approach

1. Time-consuming

2. Deterioration of facial form

3. Difficulty to chew or masticate during pre-surgical phase

4. Psychosocial problems

5. Other complications like dental decay, gum recession and root resorption or root damage

\subsection{Surgery-First Orthognathic Approach (SFOA)}

In order to overcome the shortcoming of conventional orthognathic surgery, a new approach has been conceptualized called "surgery-first orthognathic approach (SFOA)".

Even though some surgeons expressed the necessity to reposition the MMSC before performing any orthodontics in the past, it did not gain much popularity. With the advent of skeletal anchorage system in orthodontics and rigid fixation system in orthognathic surgery, the problem of skeletal relapse due to unstable bony segments during post-surgical orthodontics has been significantly reduced, paving the way for popularization of SFOA $[8,9]$.

Correction of various skeletal deformities like Class III [10] and Class II [11] and facial asymmetry [12] using SFOA technique has been reported in the past, and all of them claimed shortening of the total treatment time as a significant and greatest advantage over the conventional technique. William Bell, the "godfather" of orthognathic surgery, during one of his clinical rounds with his residents, highlighted the changing trends in orthognathic surgery by contending that the present orthognathic surgical procedures remain too complicated, too invasive, too time-consuming, too expensive, and too unpredictable, and he literally set the tone for discussing "paradigm shifts in orthognathic surgery" [13]. After the clinical applications of advanced three-dimensional imaging and office-based surgery, the 2011 symposium on SFOA created a broader interest in complete elimination of time-consuming pre-surgical reverse orthodontics and paved the way for worldwide acceptance of this paradigm shift in orthognathic surgery $[14,15]$.

SFOA technique is fundamentally a "face-first" approach wherein the patient's chief complaint is taken care immediately by improving the facial soft tissue profile and thereby increasing the patient's compliance to overall treatment (Fig. 67.1). In SFOA, greater part of the dentition remains at the same position with respect to their respective arches as there is no pre-surgical orthodontic phase involved. Following the orthognathic surgery, MMSC will be in a Class I relationship, yet the upper and lower dentition may not fit perfectly into occlusion. A treatable malocclusion, called transitional occlusion, is set at the end of surgery which can be corrected by the orthodontists. Hence, the
First - Face Approach:

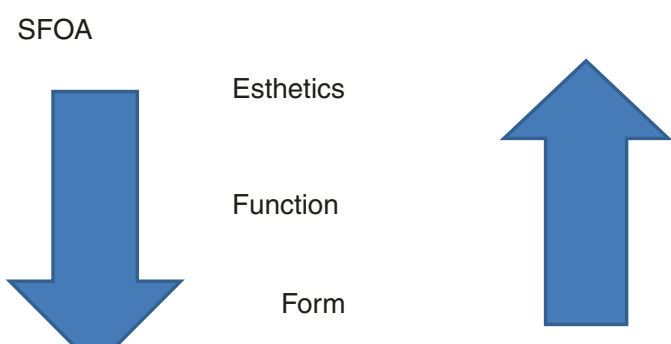

Conventional Approach

CAssociation of Oral and Maxillofacial Surgeons of India

Fig. 67.1 The flow diagram demonstrating fundamental differences and the order of priority between the SFOA and conventional approach

\section{Comparison between Conventional technique and SFOA}

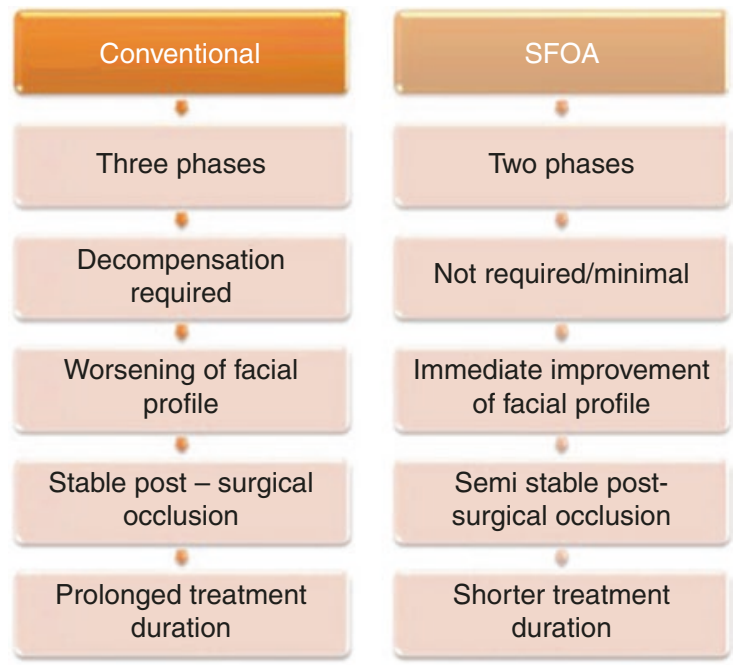

Fig. 67.2 The comparison chart demonstrating various steps for conventional technique and SFOA

orthodontist must actively participate in the treatment planning in SFOA so as to achieve a treatable transitional occlusion at the end of surgery. The orthodontist must be able to foresee the resulting occlusion even before the surgery, and this ability to plan and foresee the resultant transitional occlusion accurately is the linchpin of the entire SFOA technique. Therefore, in SFOA technique the orthodontic principles and guidelines must be established in advance so as to ensure that treatable malocclusions are attained.

Kim JH et al. [8] in their treatise called "Guidelines for "surgery first" orthodontic treatment" have emphasized caution when embarking on SFOA technique. The entire planning process (Fig. 67.2) is laborious since the skeletal changes should be preconceived and the casts should be mounted accordingly to determine the anticipated postoperative orthodontic teeth movements, and, even for the highly accomplished orthodontic and surgical team, it is 
challenging to pinpoint accurately the occlusal relationship that will balance ideal aesthetic and functional results. It should be remembered during planning that surgical movement should be adequate enough such that dental decompensation is possible after the surgery. Customarily, the dentition is bonded/banded and a passive arch wire is placed before surgery. Active orthodontic tooth movement begins within a relatively short period of time after the jaw(s) are repositioned to take advantage on the inherent phenomena called accelerated tooth movement [8].

\subsection{Regional Accelerated Phenomena (RAP)}

It is observed that the orthodontic tooth movement following orthognathic surgery is rapid and it is significantly rapid during initial 4-5 post-operative months. This rapid tooth movement post-surgery is attributed to RAP, and it is observed with all the three planes, sagittal, vertical and transverse.

It is an established fact that orthodontic tooth movement is a metabolic event of alveolar bone resorption in the areas of pressure and bone formation in the areas of tension [16, 17]. In order to analyse post-surgical changes in bone metabolism after orthognathic surgery and the corresponding response in the dento-alveolus, Liou et al. conducted a prospective clinical pilot study in 2011. All 22 adult patients who participated in their study underwent both maxillary LeFort I osteotomy and mandibular bilateral sagittal split osteotomy for repositioning of their jaws. Each of these patients was evaluated for serum alkaline phosphatase (ALP) and C-terminal telopeptide of type I collagen (ICTP) before and after surgery at sequential intervals for up to 4 months post-operatively [14].

ALP and ICTP are two bone markers which are indicative of bone turnover rate. ICTP is a bone resorption metabolite of type I collagen in bone and has been associated with the activities of osteoclasts [18]. ALP is an enzyme for bone formation and has been found to associate with osteoblasts function [19]. The results of Liou et al.'s study [14] showed transient increase in the levels of both ICTP and ALP in the immediate post-surgical period indicating burst in the activity of bone remodelling and turnover activity. It was observed from Liou et al.'s [14] study that the ICTP levels peaked from 1 week to 3 months and the ALP levels from 1 to 4 months post orthognathic surgery. This increase in bone turnover markers in the initial post-operative period is a clear indicator of high metabolic activity in the bone and therefore can be used to advantage in shortening the total treatment time in SFOA.

The RAP is not isolated to SFOA. Even in conventional, orthodontics-first approach, this phenomenon plays its role in post-surgical orthodontics. The only difference is that in conventional technique the surgery is done after the decompensation is achieved. The SFOA technique utilizes the golden opportunity of RAP by speeding up the decompensation process which occurs after the orthognathic surgery contrary to that in conventional technique. Therefore, in SFOA, the surgical procedure increases the bone metabolic rate of dento-alveolus in the first 4 post-operative months, which in turn accelerates the orthodontic tooth movement. Hence to make complete use of RAP in SFOA, the post-surgical orthodontic tooth movements have to be begun as early as $1 \mathrm{st}$ week and completed by the 4th month.

\subsection{Indications}

Though SFOA technique can be employed in any kind of maxillomandibular skeletal discrepancy, Liou et al. proposed the following indications in 2011 (Box 67.1) which could help in easy case selection and will act as a guide for the beginners.

\subsection{Treatment Planning Considerations}

\subsubsection{General Guidelines}

- The upper and lower dentition are bonded and banded before the surgery without placing any arch wire in order to keep the dentition undisturbed and solid during and after the surgery.

- When planning for SFOA, multiple treatment options must be taken into account. It is very important for the orthodontist to plan the resulting occlusion on the preoperative model set-up in order to achieve relatively stable occlusion on the table during surgery.

- The decompensation of the teeth to normal positions and angulations will be done after surgery, and hence transitional occlusion should allow any orthodontic teeth movement after the surgery. Since the incisors cannot be used as a guide to foresee the final form of occlusion in SFOA, the molar relationship can be utilized as a starting point to come up with a temporary occlusion.

Box 67.1 Proposed indications for SFOA technique

- Well-aligned to mildly crowded anterior teeth

- Flat to mild curve of Spee

- Normal to mildly proclined/retroclined incisors

- Minimal transverse discrepancies

- Cases in which decompensation is not required or minimal decompensation is required 
- For the model surgery, the maxillary and mandibular casts are positioned in a proper molar relationship and with a positive overbite. The molar relation could be set up in Class I in cases of non-extraction or bimaxillary first premolar extraction, Class III in cases of lower first premolar extraction and Class II in cases of maxillary first premolar extraction. Once the molar relationship has been established, the overjet should also have been determined.

- The inclination and angulations of upper incisors determine the pattern of extractions. If upper incisors are excessively inclined, then premolar extraction needs to be planned in order to allow post-operative retraction of upper incisors. As a rule of thumb, if the upper incisor to occlusal plane angulation is less than $53-55^{\circ}$, extraction must be considered [20].

- The transverse dimension often creates a special challenge when performing model surgery in surgery-first cases. The upper and lower midlines must be coincident or close to it post-surgery, and proper buccal overjet must be established bilaterally.

- Once surgery is completed, the post-operative orthodontic treatment can begin anytime between 1 week and 1 month post-operatively in order to take the advantage of RAP.

Envisioning the final dental occlusion based on the present occlusion is the key factor in success of SFOA. The interim transitional occlusion achieved on preoperative model set-up must be stable enough to allow predictable splint preparation and skeletal movement. It is highly recommended that at least three point contacts are achieved between upper and lower teeth when planning for interim transitional occlusion. In situations where such transient occlusion cannot be established, it is advisable to commence some orthodontic movement in order to assuage some of the interferences and allow for a more stable transitional malocclusion to be established.

\subsubsection{Specific Guidelines [15]}

\section{Anteroposterior and Vertical Decompensation in Class III Cases}

- The sagittal decompensation for proclined upper incisors in a Class III case could be achieved by an anterior segmental osteotomy with removal of the maxillary first bicuspids or by clockwise rotation of the maxilla by LeFort I osteotomy to upright the maxillary incisor inclination.

- The anteroposterior decompensation for moderately retroclined and crowded lower incisors in a Class III case could be achieved by setting up the molars in a Class I relationship with an excessive incisor overjet, and then the mandibular incisors could be aligned after the surgery to obtain the desired overjet.
- The sagittal decompensation for severely retroclined and crowded lower incisors in a Class III situation could be achieved by extraction of the lower first bicuspid and anterior segmental osteotomy, setting up the molars in a Class III relationship with an excessive incisor overjet, and then the lower incisors could be aligned after the surgery to obtain a desired overjet.

- A moderate to deep mandibular curve of Spee in a Class III case is better levelled preoperatively or surgically by anterior segmental osteotomy to avoid the upward and forward rotation of the mandible post-operatively. Alternatively, the lower incisors could be intruded, and the upper incisors at the same time could be extruded post-operatively.

\section{Anteroposterior and Vertical Decompensation in Class II Cases}

- For a moderate to deep mandibular curve of Spee and proclined mandibular incisors in Class II mandibular retrognathism, the anterior segment of the lower jaw could be levelled and intruded surgically through anterior segmental osteotomy so that the mandible could be advanced accurately.

- Alternatively, the mandible could be surgically advanced to an edge-to-edge incisor relationship and without occlusal contact in the posterior teeth, and then post-surgically, the lower anterior teeth could be orthodontically intruded so that the mandible rotates upwards and forwards for posterior occlusal contact and a better chin projection.

\subsection{Treatment Protocol (Table 67.2)}

The protocol variations between the conventional orthognathic surgery and SFOA can be clearly appreciated in the Table 67.2 highlighted in Bold fonts. A simulation of pre-

Table 67.2 Treatment protocol comparing SFOA technique to conventional technique

\begin{tabular}{|c|c|}
\hline Conventional technique & SFOA technique \\
\hline 1. Initial diagnosis & 1. Initial diagnosis \\
\hline 2. Surgical planning-STO & 2. Surgical planning-STO \\
\hline \multirow[t]{2}{*}{$\begin{array}{l}\text { 3. Pre surgical orthodontic } \\
\text { treatment }\end{array}$} & $\begin{array}{l}\text { 3. Simulation of pre-surgical } \\
\text { orthodontic treatment }\end{array}$ \\
\hline & $\begin{array}{l}\text { Model mounting and model setup } \\
\text { 4. Simulation of orthognathic } \\
\text { surgery }\end{array}$ \\
\hline 4. Surgical arch wire & 5. Surgical arch wire \\
\hline 5. Fabrication of splints & 6. Fabrication of splints \\
\hline $\begin{array}{l}\text { 6. Orthognathic surgery and } \\
\text { post-op care }\end{array}$ & $\begin{array}{l}\text { 7. Orthognathic surgery and } \\
\text { post-op care }\end{array}$ \\
\hline 7. Orthodontic rediagnosis & 8. Orthodontic rediagnosis \\
\hline 8. Orthodontic treatment & 9. Orthodontic treatment \\
\hline 9. Finishing & 10. Finishing \\
\hline
\end{tabular}


surgical orthodontic treatment using model mounting and set-up will compensate the pre-surgical orthodontic treatment.

\subsection{Protocol Variations}

Although the sequence of treatment is similar, different protocols are being used to prepare the patient for surgery, perform the operative procedure, and commence the orthodontic treatment.

\subsubsection{Timing of Bonding}

- Chung C Yu and Villegas [12]_-1 week before orthognathic surgery

- Sugawara and Nagasaka [10, 11]; E Liou et al. [15]—Just before surgery

- Federico Hernandez [21]—brackets 10-14 days after surgery

\subsubsection{Initial Arch Wires}

- Liou et al. [15] — did not place any orthodontic arch wires before surgery

- Sugawara and Nagasaka [10, 11]-preferred $0.019^{\prime \prime} \times 0.025^{\prime \prime} \mathrm{SS}$ wires in $0.022^{\prime \prime}$ slot

Placement of passive stainless steel arch wires bent and adapted to each tooth before surgery will preclude any untoward tooth movement during or immediately after the surgery. The authors wishing to capitalize on the RAP concept immediately after surgery prefer to place active nickeltitanium arch wires prior to surgery. Nonetheless, in doing so, the orthodontist loses an opportunity to observe the stability of the surgical correction prior to beginning of the tooth movement. The rapid acceleratory phenomenon not just influences the tooth movement but also can disturb the alveolar bone [8].

There are also protocol variations regarding the usage of surgical splints after the surgery among the various authors. While some authors suggested the use of the surgical splints only intraoperatively, others have advocated its use anywhere between 1 and 4 post-operative weeks. Nagasaka et al. have used removable Gelb-type splints post-surgery [10]. Kim's preference is to leave the splint in for about 1 month to 1 and a half months post-operatively and if an open bite is observed to use elastics between the splint and the mini-screws placed into the bone or to leave the splint for an even longer time period [8].

\subsection{Procedural Guidelines for Model Mounting and Model Set-Up in SFOA} (Fig. 67.3)

- Once the treatment planning and diagnosis is completed, impressions are made and customary model mounting is performed with bite registration to scrutinize the present state of the occlusion.

- In the model set-up, teeth that have adapted by natural compensation to the existing skeletal discrepancy are simulated and reorganized into anticipated occlusion similar to the preoperative orthodontic treatment plan. All the teeth on the set-up model are rearranged as if orthodontic treatment is done in the real patient in conventional orthognathic surgery technique into the desired preoperative occlusal relationship.

- Once the desired dental occlusion is set on the models, the amount of the skeletal movement required in the maxilla, mandible or both will become evident. Subsequently, a simulation of the actual orthognathic surgery is performed on the mounted models as in conventional technique. This will indicate the possible occlusal outcome of the standard approach.

- Intermediate and final splint can then be made on these mounted models as they are set up into the planned skeletal movements.

- With the advent of virtual planning and three-dimensional simulation softwares, the same set-up model surgery can be performed by scanning the physical models and bite registration details into the software, and the three-dimensional intermediate and final splints can be printed.

\subsection{Surgical Procedures (Fig. 67.4)}

The surgical procedures in the maxilla, mandible or chin are themselves performed using described standard techniques, whether it is the conventional technique or the SFOA technique. 

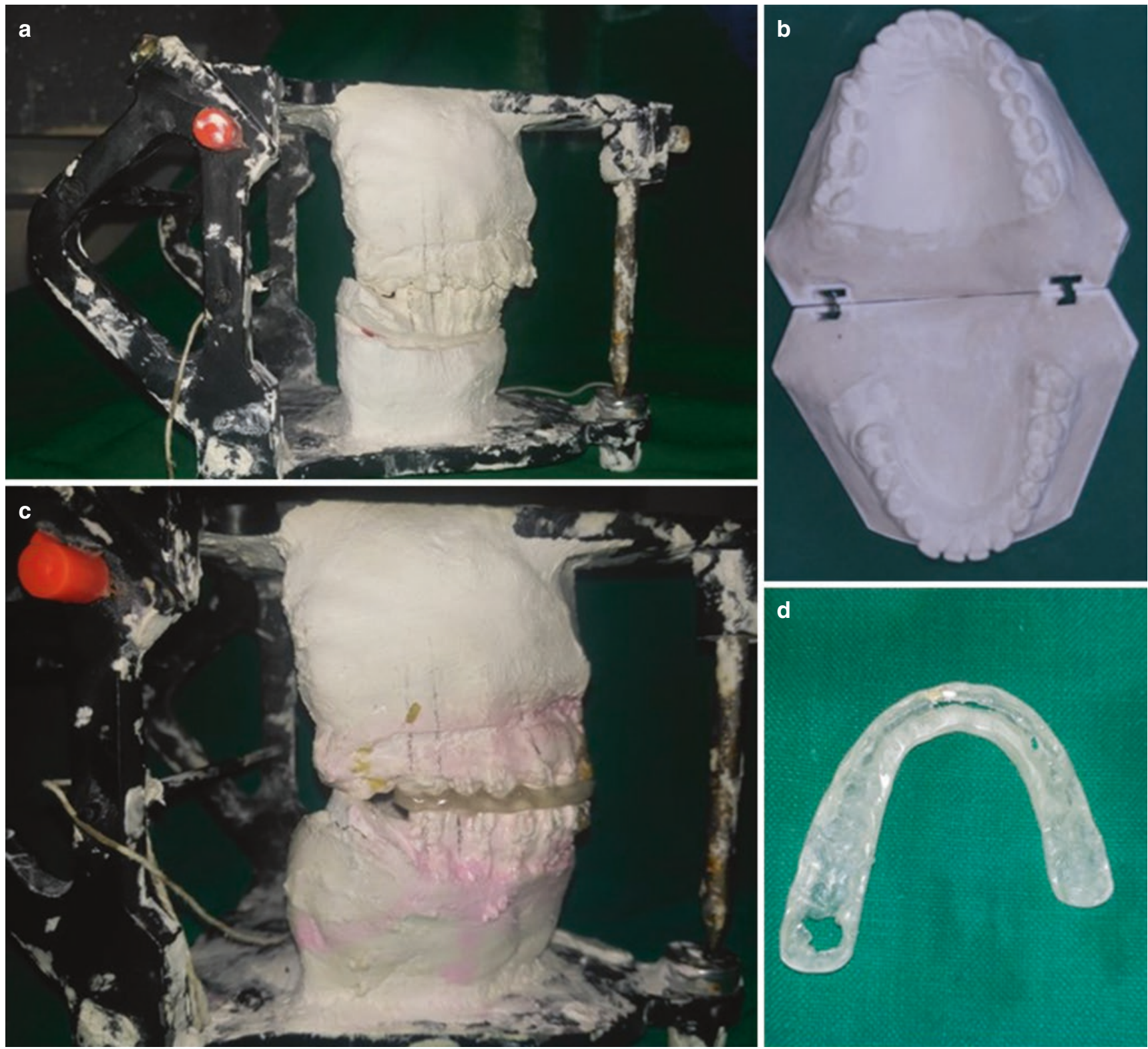

CAssociation of Oral and Maxillofacial Surgeons of India

Fig.67.3 (a) Standard model mounting. (b) Before the procedure, the teeth that have adapted to the skeletal discrepancy are simulated and reorganized into their predicted location, similar to real pre-surgical

Fig. 67.4 Flow diagram showing various surgical procedures in each of the jaws orthodontic treatment. (c) Simulation of actual orthognathic surgery is then performed. (d) Surgical splints fabricated to aid in real-time surgery
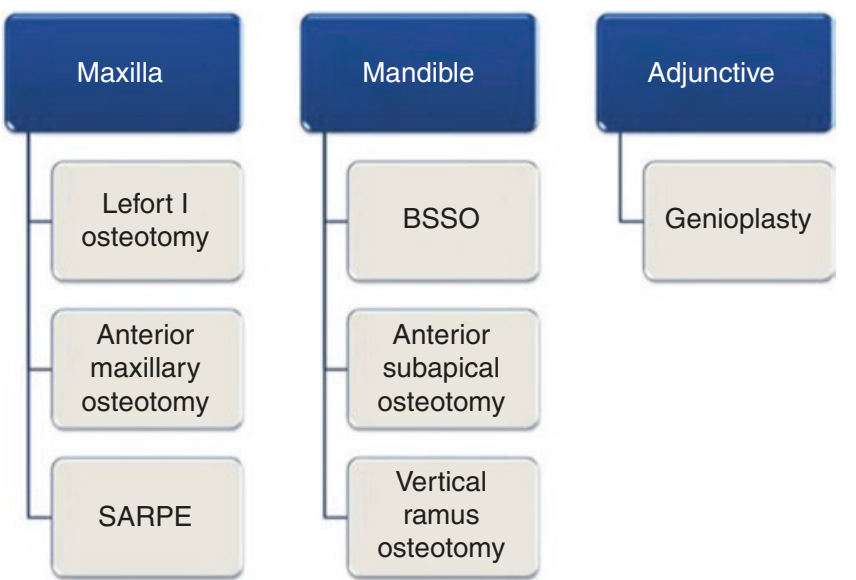

CAssociation of Oral and Maxillofacial Surgeons of India 


\subsection{Advantages and Disadvantages of SFOA (Table 67.3)}

Systematic review published by Peiró-Guijarro et al. [21] in 2006 reviewed 179 publications and finally selected 11 articles for final reporting. They found that the SFOA technique was most commonly reported in Class III skeletal malocclusion than in skeletal Class II malocclusion with prevalence of about $84.7 \%$. The single largest advantage described was shortened treatment duration time owing to the RAP concept, and the disadvantage was potential error in predicting the post-operative occlusal outcome resulting in greater or lesser surgical movements of the jaws.

\subsection{The Future of "Surgery-First" Approach}

The future of SFOA technique lies in using augmented skull models, virtual orthodontic set-up to replace the mounted study model set-up and the computer-aided design and computer-aided manufacturing fabrication of intraoperative splints (Fig. 67.5). The 3D virtual orthodontic set-up will help the orthodontist to predict the eventual (at the end of orthodontic treatment) position and the axial inclination of each tooth scrupulously. This is a compelling step before the surgeon's skeletal base correction simulation, since the patient's prevailing occlusion

Table 67.3 Advantages and disadvantages of the surgery-first approach

\section{Advantages}

1. Total duration of treatment is shorter

2. The facial profile is improved from the commencement of treatment as a result of skeletal base correction

3. Patient and orthodontist satisfaction rates are high. High patient satisfaction is associated with improved cooperation during post-operative orthodontics

4. Orthodontic decompensation is efficient and effectual in response to the establishment of a proper maxillomandibular relationship and the regional acceleratory phenomenon

5. Surgical movements may not be curbed due to orthodontic constraints. There is more freedom to choose the surgical movement in accordance with the clinical requirement for individual patients

6. Patient recovery takes place expeditiously

7. When sleep-disordered breathing is the main indication for treatment, early maxillomandibular advancement increases the dimensions of the upper airway immediately

Disadvantages

1. Case selection is critical because the baseline occlusion cannot guide treatment objectives. Therefore, high clinical expertise, meticulous prediction of post-operative tooth movement and precise assessment of skeletal discrepancy are imperative

2. The bending procedure for a passive surgical wire is tedious and complex

3. Bonding and removal of the surgical wire are taxing; there is a relatively high bonding failure rate before and during surgery

4. The extent of surgical movements is necessarily greater, because surgical correction needs to make up for dental compensation

5. Impacted lower third molars could add difficulty to surgery

6. ost-surgical fluidity during bone healing could cause skeletal instability, and its impact on relapse has not yet been fully investigated

7. Orthodontic appointments should be scheduled more often than in a traditional approach. This could be stressful for the orthodontist

8. Constant correspondence between the surgeon and the orthodontist is indispensable
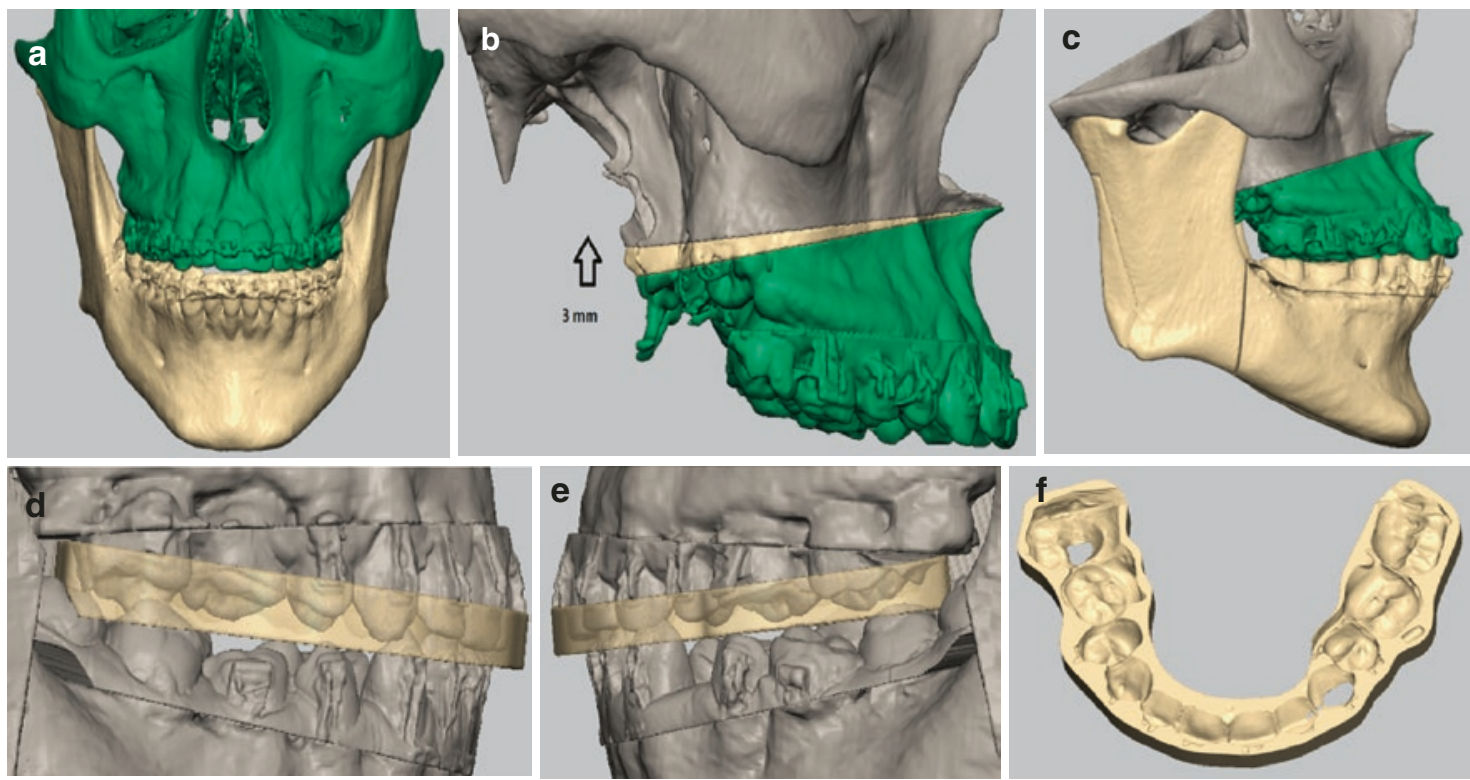

(C)Association of Oral and Maxillofacial Surgeons of India

Fig. 67.5 The workflow demonstrating the pre-surgical orthodontic movement set-up (a), the surgical procedure on the maxilla (b), the procedure on the mandible (c), the post-operative ensuing dental occlusion with splint in situ (d, e) and the design of the final splint (f) 
cannot serve as a guide for skeletal repositioning as it lies in a compensated state.

Usability of temporary anchorage devices (TAD) and interdental corticotomies is a factor which can add to the future of SFOA. The TAD permits a wider range of orthodontic vectors and avoids premature bracket loading with secondary troublesome dental extrusion. Interdental corticotomies can augment the RAP and further enhance the orthodontic treatment duration.

\subsection{Conclusion}

Performing orthognathic surgery even before any orthodontic tooth movement (SFOA) offers a unique advantage of addressing the patient's chief complaint at the very beginning, thereby improving the acceptance and compliance of the patient to overall treatment. It also offers the big advantage of significant decrease in total duration time by making use of the RAP. The final outcomes, in the way of facial aesthetics, dental occlusion and stability, are similar when using orthodontics-first and surgery-first approaches. However, it must be remem- bered that both the surgeon and the orthodontist must trend with care as there is a premium on patient selection and both should be involved as a team during every stage of the treatment, starting from diagnosis to debonding. Surgeonorthodontist team should know the orthodontic principles and understand the limits of orthodontic teeth movement and must accommodate dental decompensation in their initial treatment planning. The surgeon should be capable of carrying out designated osteotomy and intermaxillary fixation with occlusion bite plate on misaligned dental arches and providing the stability after skeletal reposition. The future of orthognathic surgery is geared towards reducing the overall treatment duration without compromising the final outcome.DisclosureAuthors have no financial conflicts to disclose.

\subsection{Case Scenarios}

\section{Case Scenario 1 (Fig. 67.6)}

\section{Case Scenario 2 (Fig. 67.7)}
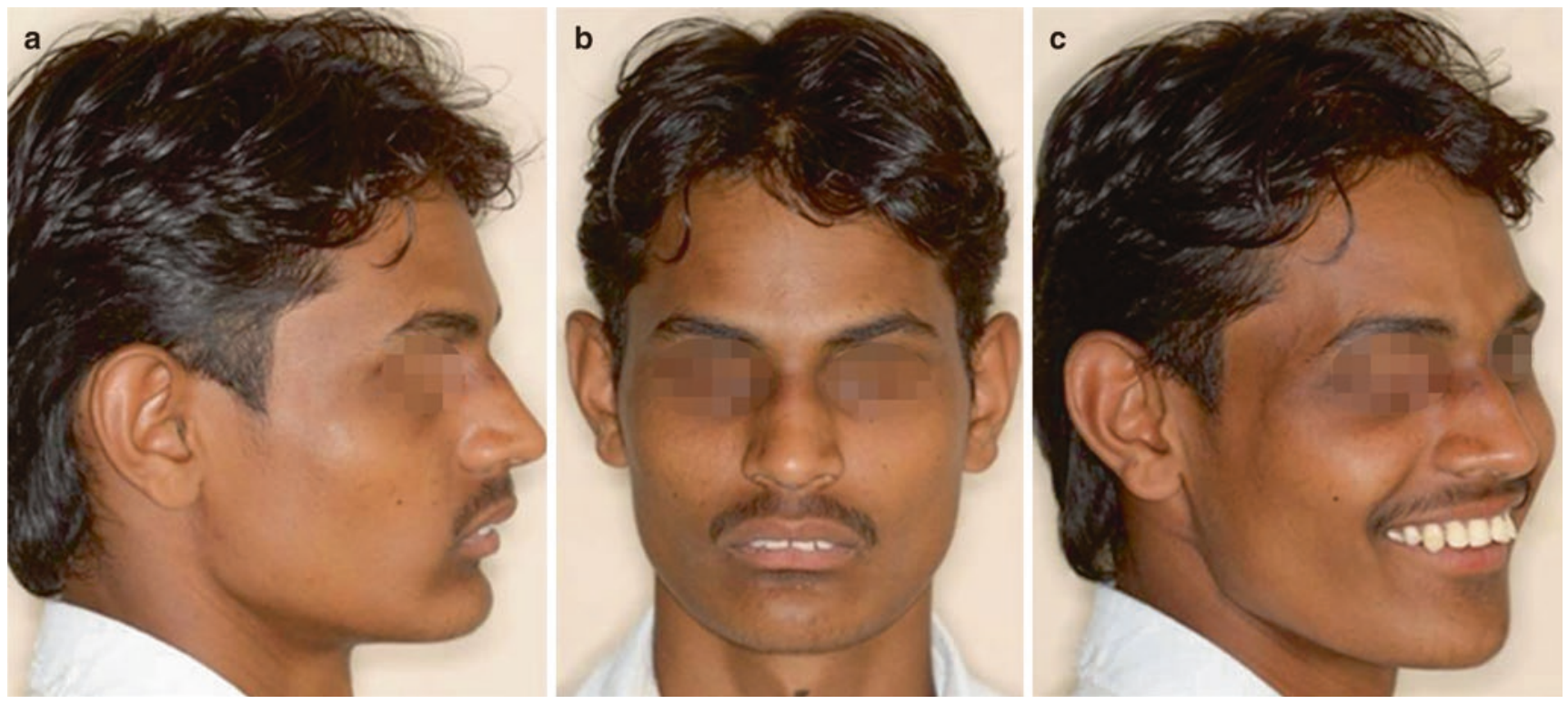

Fig. 67.6 Case Scenario 1. A 19-year-old male presenting with forwardly place upper front teeth. (a-c) Preoperative pics showing frontal, profile and right lateral views. (d, e) Preoperative occlusion showing end on molar relation and compensated dental occlusion with anterior deep bite and excessive overjet. (f, g) Post-surgical orthodontic treat- ment in progress. Excessive nature of curve of Spee can be noted, and the change in overjet and overbite post-surgery can be appreciated. (hj) Post-treatment facial pics: frontal, smiling and lateral views. (k, l) Post-treatment intraoral pics. $(\mathbf{m}, \mathbf{n})$ Comparison of pre-operative and post-operative lateral cephalograms 

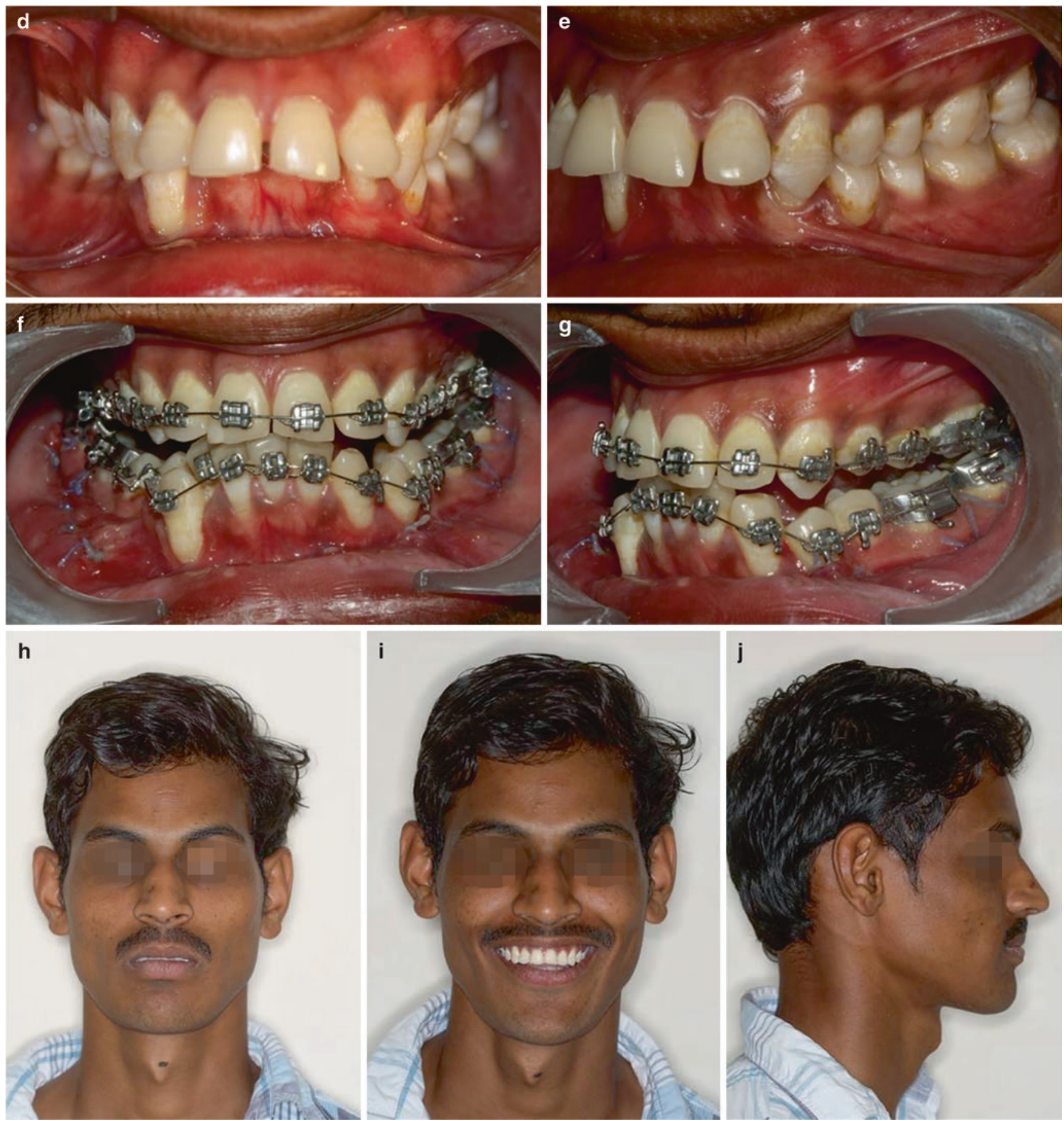

Fig.67.6 (continued) 

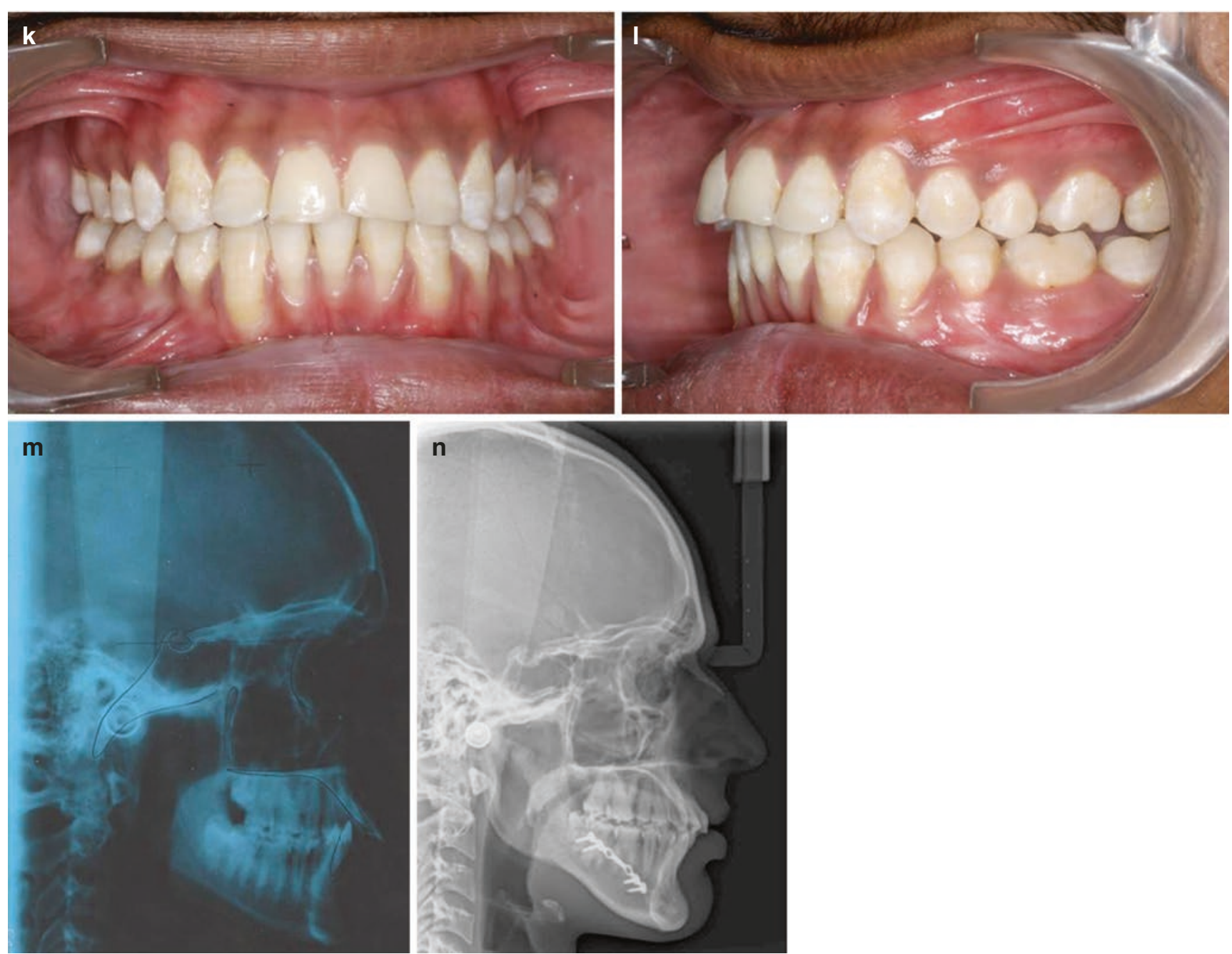

(CAssociation of Oral and Maxillofacial Surgeons of India

Fig. 67.6 (continued) 

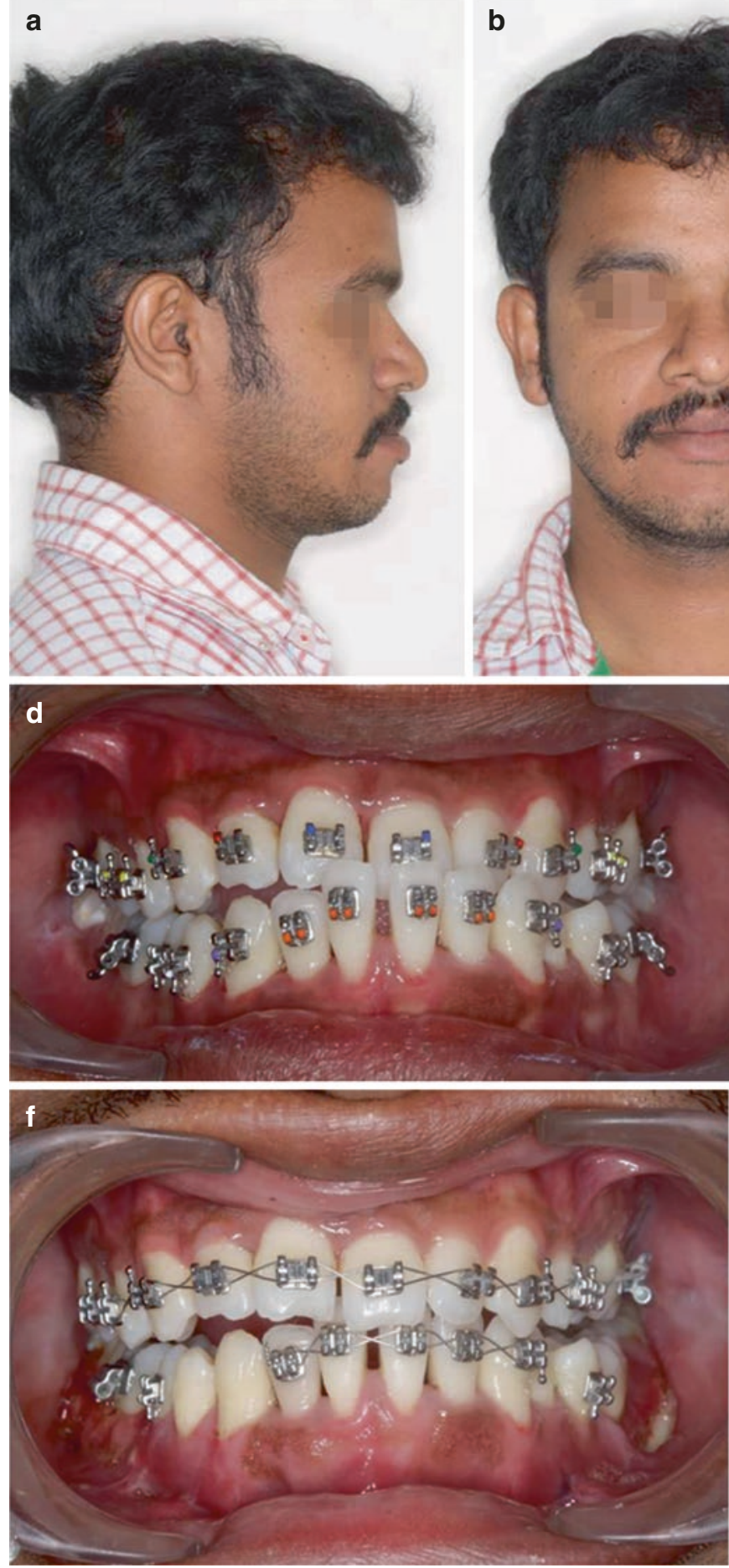

Fig. 67.7 Case Scenario 2. A 26-year-old male presenting with prominent lower facial appearance and mobile lower front teeth. (a, b) Clinical pics showing lateral and frontal views: Concave facial profile can be appreciated with acute nasolabial angle. (c) Lateral cephalogram showing skeletal Class III jaw bases with normodivergence. (d, e) Intraoral picture showing reverse overjet, Class III molar relationship
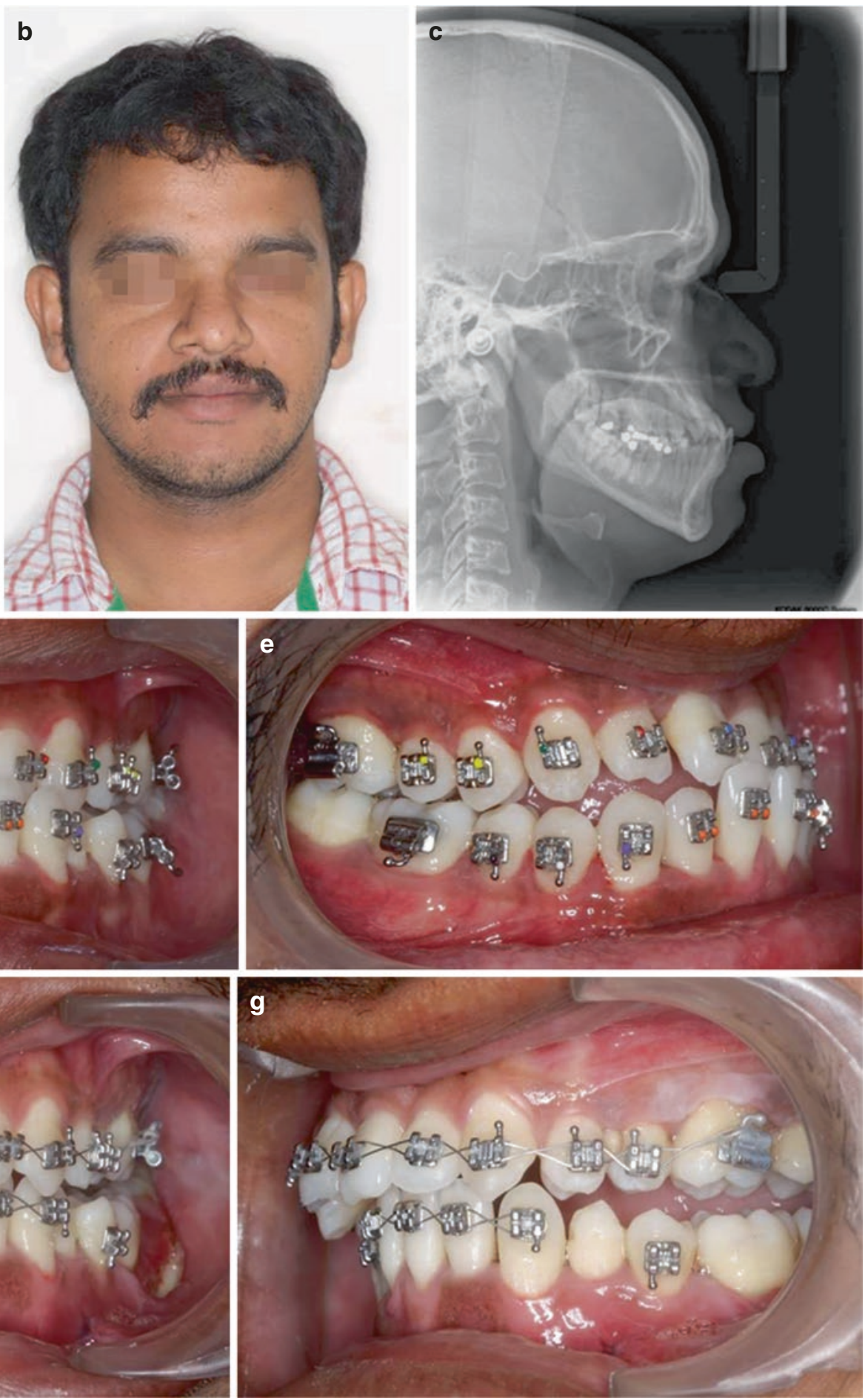

and spacing in lower anteriors. (f, g) Post-surgical orthodontics in progress. Reversal of overjet can be noticed. (h-J) Post-treatment facial pics in profile and frontal. (k) Intraoral post-treatment pic showing occlusal relationship with normal overjet and Class I molar relationship. (l) Postoperative cephalogram 

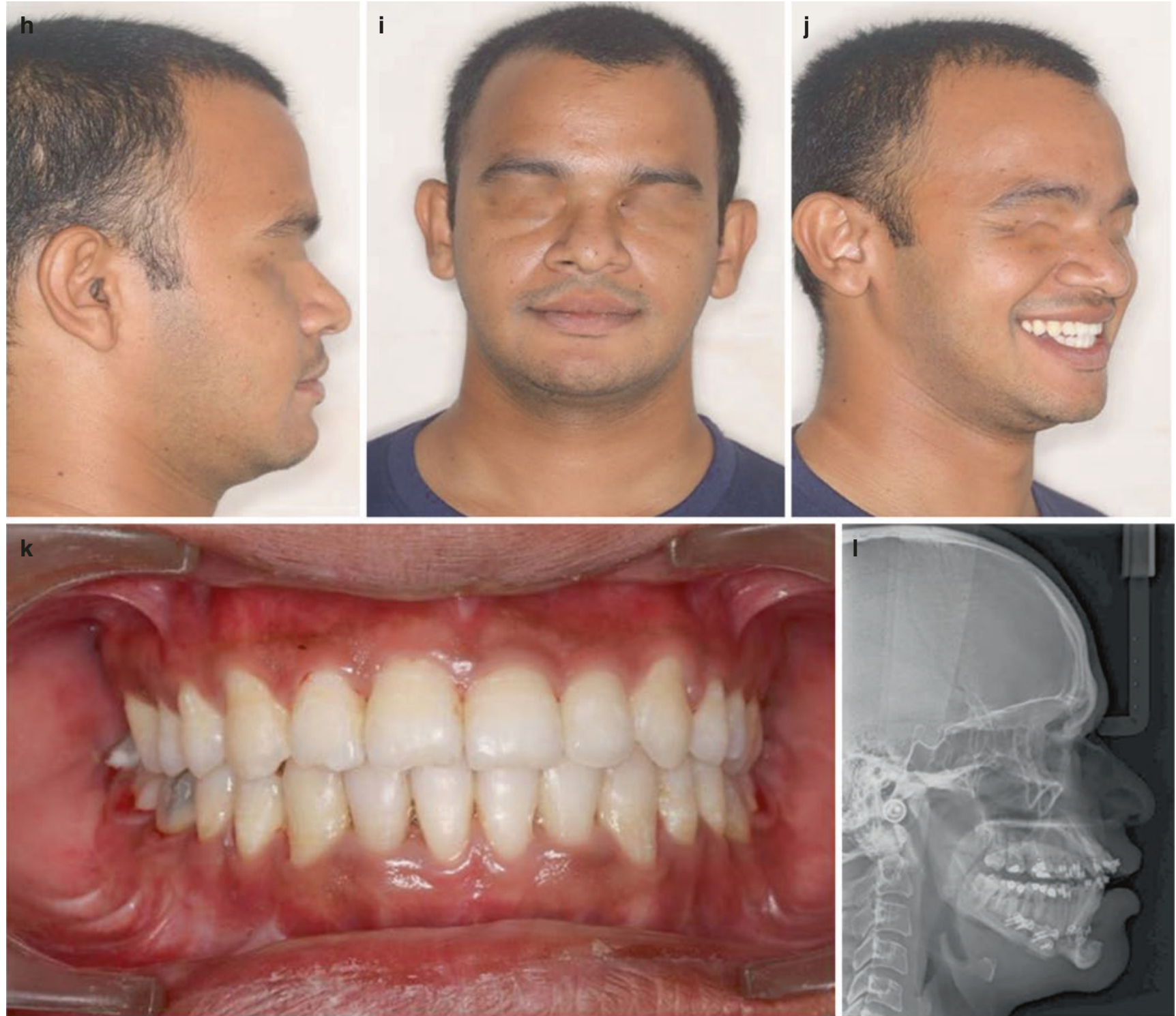

CAssociation of Oral and Maxillofacial Surgeons of India

Fig. 67.7 (continued)

\section{References}

1. Poulton DR, Taylor RC, Ware WH. Cephalometric x-ray evaluation of the vertical osteotomy correction of mandibular prognathism. Oral Surg Oral Med Oral Pathol. 1963;16:807-20.

2. Worms FW, Isaacson RJ, Speidel TM. Surgical orthodontic treatment planning: profile analysis and mandibular surgery. Angle Orthod. 1976;46(1):1-25.

3. Sabri R. Orthodontic objectives in orthognathic surgery: state of the art today. World J Orthod. 2006;7(2):177-91.

4. Grubb J, Evans C. Orthodontic management of dentofacial skeletal deformities. Clin Plast Surg. 2007;34(3):403-15.

5. Bailey LTJ, Proffit WR. Combined surgical and orthodontic treatment. In: Sarver DM, Fields HW, Proffit WR, editors. Contemporary orthodontics. 3rd ed. Philadelphia, PA: Mosby; 1999. p. 674-709.

6. Jacobs JD, Sinclair PM. Principles of orthodontic mechanics in orthognathic surgery cases. Am J Orthod. 1983;84(5):399-407.
7. Proffit WR, White RP, Sarver DM. Combining surgery and orthognathics: who does what, when? In: Proffit WR, White RP, Sarver DM, editors. Contemporary treatment of dentofacial deformity. 1st ed. St Louis, MO: Mosby; 2003. p. 245-67.

8. Kim JH, Mahdavie NN, Evans CA. Guidelines for "surgery first" orthodontic treatment. In: Bourzgui F, editor. Orthodonticsbasic aspects and clinical considerations. 1st ed. Casablanca: University Hassan II of Casablanca: IntechOpen; 2012. p. 265-300.

9. Huang CS, Chen YR. Orthodontic principles and guidelines for the surgery-first approach to orthognathic surgery. Int J Oral Maxillofac Surg. 2015;44(12):1457-62.

10. Nagasaka H, Sugawara J, Kawamura H, Nanda R. "Surgery first" skeletal class III correction using the skeletal anchorage system. J Clin Orthod. 2009;43(2):97-105.

11. Sugawara J, Aymach Z, Nagasaka DH, Kawamura H, Nanda R. "Surgery first" orthognathics to correct a skeletal class II malocclusion with an impinging bite. J Clin Orthod. 2010;44(7):429-38. 
12. Villegas C, Uribe F, Sugawara J, Nanda R. Expedited correction of significant dentofacial asymmetry using a "surgery first" approach. J Clin Orthod. 2010;44(2):97-103.

13. Assael LA. The biggest movement: orthognathic surgery undergoes another paradigm shift. J Oral Maxillofac Surg. 2008;66(3):419-20.

14. Liou EJ, Chen PH, Wang YC, Yu CC, Huang CS, Chen YR. Surgery-first accelerated orthognathic surgery: postoperative rapid orthodontic tooth movement. J Oral Maxillofac Surg. 2011;69(3):781-5.

15. Liou EJ, Chen PH, Wang YC, Yu CC, Huang CS, Chen YR. Surgery-first accelerated orthognathic surgery: orthodontic guidelines and setup for model surgery. J Oral Maxillofac Surg. 2011;69(3):771-80.

16. Storey E. The nature of tooth movement. Am J Orthod. 1973;63(3):292-314.
17. Reitan K. Some factors determining the evaluation of forces in orthodontics. Am J Orthod. 1957;43(1):32-45.

18. Pellegrini GG, Gonzales CM, Somoza JC, Friedman SM, Zeni SN. Correlation between salivary and serum markers of bone turnover in osteopenic rats. J Periodontol. 2008;79(1):158-65.

19. Binder T, Goodson J, Socransky S. Gingival fluid levels of acid and alkaline phosphatase. J Periodontal Res. 1987;22(1):14-9.

20. Liao YF, Chiu YT, Huang CS, Ko EW, Chen YR. Presurgical orthodontics versus no presurgical orthodontics: treatment outcome of surgical-orthodontic correction for skeletal class III open bite. Plast Reconstr Surg. 2010;126(6):2074-83.

21. Peiró-Guijarro MA, Guijarro-Martínez R, HernándezAlfaro F. Surgery first in orthognathic surgery: a systematic review of the literature. Am J Orthod Dentofac Orthop. 2016;149(4):448-62.

Open Access This chapter is licensed under the terms of the Creative Commons Attribution 4.0 International License (http://creativecommons. $\mathrm{org} /$ licenses/by/4.0/), which permits use, sharing, adaptation, distribution and reproduction in any medium or format, as long as you give appropriate credit to the original author(s) and the source, provide a link to the Creative Commons license and indicate if changes were made.

The images or other third party material in this chapter are included in the chapter's Creative Commons license, unless indicated otherwise in a credit line to the material. If material is not included in the chapter's Creative Commons license and your intended use is not permitted by statutory regulation or exceeds the permitted use, you will need to obtain permission directly from the copyright holder. 